\title{
Modification of LDPE molecular structure by gamma irradiation for bioapplications
}

\author{
L.M. Ferreira $^{\mathrm{a}, *}$, A.N. Falcão ${ }^{\mathrm{a}}$, M.H. Gil ${ }^{\mathrm{b}}$ \\ a ITN, Nuclear and Technological Institute, EN 10, 2686-953 Sacavém, Portugal \\ ${ }^{\mathrm{b}}$ Department of Chemical Engineering, Faculty of Science and Technology, University of Coimbra, Pólo II - Pinhal de \\ Marrocos, 3030-290 Coimbra, Portugal
}

Available online 23 May 2005

\begin{abstract}
The surface properties of low-density polyethylene (LDPE) can be modified by the grafting of 2-hydroxyethyl methacrylate (HEMA). This was done aiming at the production of new materials suitable for bioapplications. Samples with different monomer concentrations were prepared from LDPE particles by gamma irradiation, following different irradiation protocols, including irradiation in presence and absence of air. The samples were characterized by thermal analysis techniques (DSC and TGA) and by Fourier transform infrared spectroscopy (FTIR). The results obtained show a decrease in the crystallinity of the supporting matrix for copolymers with high yields of grafting. However, the new materials prepared maintain good structural order resulting from the protective effect of polyHEMA grafted onto LDPE backbone. These effects can improve the diffusion of other species deeper inside the matrix and increase the material hydrophilicity. The studies performed made possible the selection of experimental protocols adequate for the production of new copolymeric materials with high grafting yield. These were used in the production of new LDPE films with enhanced hydrophilic properties.
\end{abstract}

(c) 2005 Elsevier B.V. All rights reserved.

PACS: $82.35 . \mathrm{Jk} ; 82.50 . \mathrm{Kx} ; 81.05 . \mathrm{Lg} ; 82.35 . \mathrm{Lr}$

Keywords: Graft copolymers; Gamma radiation; Polymer characterization; Polymers for bioapplications

\section{Introduction}

* Corresponding author. Tel.: +351 21994 6066; fax: +351 21 9941525.

E-mail address: ferreira@itn.pt (L.M. Ferreira).
In recent years there has been a growing interest in the use of polymers in the development of materials for bioapplications. However, 
only in a few cases the properties required for those applications are characteristic of the polymeric material. Graft copolymerisation induced by gamma radiation is a technique that provides a general method for the modification of physical, chemical and mechanical properties of polymers [1,2]. By controlling the experimental conditions (monomer concentration, gamma radiation dose-rate and irradiation environment), one can tailor the final properties of the samples in order to produce new materials for specific applications.

Polyethylene (LDPE) is the polyolefin most commonly used as plastic and has been extensively used as a backbone for radiation grafting of different monomers. This is essentially due to its excellent chemical resistance, high impact strength and high gas permeability. However it is a hydrophobic material and its very low water absorption restricts its use as biomaterial.

The use of 2-hydroxyethyl methacrylate (HEMA) as a grafting monomer onto a range of polymeric substrates with the aim of producing new biomaterials has been increasingly successful. Previous studies showed that the presence of HEMA in copolymers improves the biocompatibility of the materials [2-4].

The disorder promoted in the molecular structure of PE due the grafting process, when gamma radiation is used as the energetic source for reaction initiation, can improve the diffusion coefficients of other species deep inside the polymeric matrix, increasing the material hydrophilicity.

Aiming at the production of new grafted copolymeric LDPE films, suitable to be used for bioapplications, we have prepared and characterized polyethylene-g-hydroxyethyl methacrylate (PE-g-HEMA) copolymers.

Different sample preparation protocols were tested, looking for those that could lead to final graft copolymers with high grafting yields and homogeneous distribution. Several factors may affect the radiation grafting process, and in this paper we focus on the effects of HEMA monomer concentration, of the presence or absence of air during irradiation, and of irradiation dose-rate (DR).

\section{Experimental}

\subsection{Materials}

Pellets of low-density $\left(\rho=0.918 \mathrm{~g} \mathrm{~cm}^{-3}\right)$ polyethylene (LDPE) were supplied by Borealis, Sines, Portugal. They were grinded and sieved $(\phi=$ $500 \mu \mathrm{m})$, and than purified by extraction in a Soxhlet with absolute ethanol for $6 \mathrm{~h}$. The material was then dried to constant weight under vacuum $\left(10^{-3} \mathrm{mbar}\right)$ at $40{ }^{\circ} \mathrm{C}$. LDPE bioriented film $\left(\rho=0.920 \mathrm{~g} \mathrm{~cm}^{-3}\right)$ of thickness $15 \mu \mathrm{m}$ for food use, was kindly donated by Fibope, S.A., Portugal, and used without further purification. 2Hydroxyethyl methacrylate (HEMA) was obtained from Acros Organics, purity 98\%, New Jersey, USA, and used as received. PolyHEMA was synthesized from pure HEMA solution by irradiation in the absence of air at a DR = $0.3 \mathrm{kGy} \mathrm{h}^{-1}$ for $30 \mathrm{~h}$. The irradiated material was then washed thoroughly using $\mathrm{MeOH}$, and dried in vacuum $\left(10^{-3} \mathrm{mbar}\right)$ at $40^{\circ} \mathrm{C}$. Ethanol absolute $(\mathrm{EtOH})$ and methanol $(\mathrm{MeOH})$, p.a. reagent grade, were supplied by Riedel-de Haën, Seelze, Germany.

\subsection{Radiation graft copolymerisation}

Particles of LDPE ( $2 \mathrm{~g}$ ) with a granularity of $500 \mu \mathrm{m}$ were dispersed in $30 \mathrm{~cm}^{3}$ of HEMA solutions in $\mathrm{MeOH}$. The mixtures were then gamma irradiated at a ${ }^{60} \mathrm{Co}$ source at dose-rates of 0.3 and $0.5 \mathrm{kGy} \mathrm{h}^{-1}$. Three $[\text { HEMA] }]_{i}$ were used, 5, 10 and $15 \%(\mathrm{~V} / \mathrm{V})$, respectively. Many different total doses of exposure were tested.

Samples were irradiated both in the presence and the absence of air. For the experiments in the absence of air, the samples were deaerated by bubbling pure nitrogen in the bulk heterogeneous medium, before sealing the ampoules that contained them.

After irradiation, grafted LDPE particles were filtered from the bulk solution and washed thoroughly with $\mathrm{MeOH}$. The grafted products were then extracted with $\mathrm{MeOH}$ in a Soxhlet for $4 \mathrm{~h}$ to remove the undesirable residual monomer and homopolymer, and finally dried in vacuum $\left(10^{-3}\right.$ mbar) at $40^{\circ} \mathrm{C}$. 
The procedure for the LDPE grafted films preparation was similar. Strips of LDPE bioriented film were weighed, attached to a special rigid frame in order to prevent the film from curling during irradiation, and immersed in the monomer solution in glass ampoules. The experimental conditions of irradiation were the ones that lead to high yields of grafting in the case of the LDPE particles.

The grafting yield was determined by the percentage increase in weight as follows:

Grafting yield $(\%)=\left[\left(W_{\mathrm{g}}-W_{0}\right) / W_{0}\right] \times 100$,

where $W_{0}$ and $W_{\mathrm{g}}$ represent the weights of the initial and grafted polymer, respectively.

\subsection{Samples characterization}

\subsubsection{Thermal analysis}

The thermal properties of non-irradiated and irradiated LDPE (particles and film), polyHEMA and LDPE based copolymers were evaluated by TGA and DSC. These experiments were carried out at $10^{\circ} \mathrm{C} / \mathrm{min}$ in nitrogen atmosphere from 25 to $550{ }^{\circ} \mathrm{C}$, using DuPont Instruments equipment (TGA-model 951; DSC-model 910).

\subsubsection{Fourier transform infrared spectroscopy (FTIR)}

Infrared spectra were collected using a FTIR spectrometer from Bruker (Tensor 27 CSL). Spectra of dry particle samples (LDPE, copolymers and polyHEMA) were recorded at ambient temperature in $\mathrm{KBr}$ discs. The spectra from IR films were obtained directly from the films (LDPE and PE-g-HEMA). All spectra were recorded at the resolution of $4 \mathrm{~cm}^{-1}$ (nine scans).

\subsubsection{Films thickness}

The thickness of the films was estimated using a Mitutoyo micrometer thickness gauge.

\subsubsection{Hydration capacity studies}

The hydration behaviour of the prepared LDPE grafted films was evaluated by immersion in physiological serum $(\mathrm{NaCl} 0.9 \%)$ at $37^{\circ} \mathrm{C}$. The experiments were carried out by measuring the weight gain of clean dried grafted film samples of known weight as a function of immersion time. After each immersion period the samples were removed, blotted quickly with absorbent paper and then weighed. The process was repeated until the samples reached their hydration equilibrium, attained when consecutive determinations gave the same weight.

The isothermal desadsorption studies were performed at $37^{\circ} \mathrm{C}$, on the same samples used in the hydration studies using the TGA-951 equipment from Dupont Instruments. All the samples used were first brought to their maximum hydration degree. The percent of hydration was calculated by the following expression:

Hydration $(\%)=\left[\left(W_{\mathrm{w}}-W_{\mathrm{d}}\right) / W_{\mathrm{d}}\right] \times 100$,

where $W_{\mathrm{d}}$ and $W_{\mathrm{w}}$ represent the weights of dry and wet grafted films, respectively.

\section{Results and discussion}

\subsection{Preparation of graft copolymers}

The grafting reactions induced by gamma radiation were carried out in methanol. $\mathrm{MeOH}$ has been reported to be a good solvent for HEMA and for the homopolymer, polyHEMA, thus avoiding Tromnsdorff effects [3] - no viscous solutions were obtained in all the radiation induced copolymerisations. Figs. 1 and 2 show the effect of $[\mathrm{HEMA}]_{i}$ on the grafting yield of HEMA onto LDPE (particles) at average irradiation dose-rates of 0.3 and $0.5 \mathrm{kGy} \mathrm{h}^{-1}$. Results obtained in the presence and the absence of air are shown.

The data show that: (i) the grafting yield depends on [HEMA $]_{i}$ in an almost linear way, being the highest for the highest [HEMA] $]_{i}$; (ii) dose-rate is determinant for the final grafting yield, low dose-rates being more favourable; (iii) in the presence of air, an initial induction period is observed. The presence of oxygen leads to the formation of peroxides [5], which can compete with the less reactive monomers in the access to grafting sites within the polymer [6]. Data show that this fact is more evident at low radiation doses with lower dose-rates of irradiation as expected from the longer exposure time for the same final dose received; (iv) in all cases studied the grafting yield goes through a clear maximum at a certain 

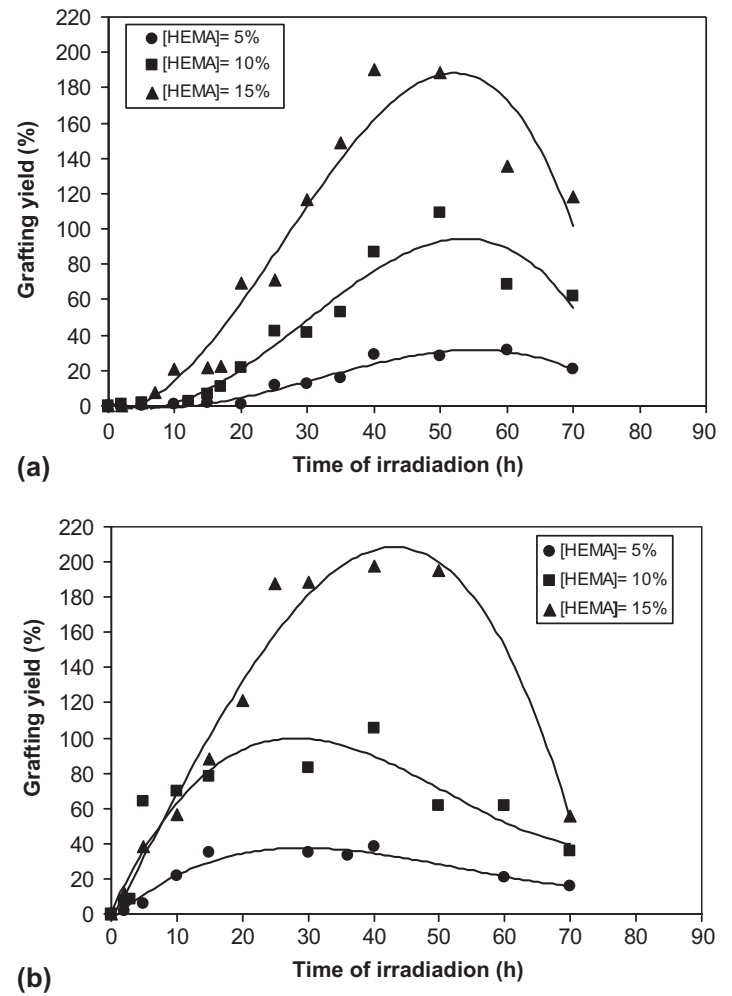

Fig. 1. Effect of [HEMA $]_{i}$ on the grafting yield of HEMA onto LDPE (particles) at an average dose-rate of $0.3 \mathrm{kGy} \mathrm{h}^{-1}$ : (a) gamma irradiation in presence of air; (b) gamma irradiation in absence of air.

irradiation dose, after which begins to decrease with the increase of total absorbed dose. This suggests that for doses above that maximum yield, scission of polyHEMA structures seems to dominate over chain linking processes. This happens at a reasonably low dose. This shape of the grafting yield versus dose curve has also been observed in other systems where HEMA was grafted to other polymeric substrates. The effect will be addressed in a forthcoming paper.

LDPE films were grafted at $14.0 \%$ and $268.0 \%$ using a $[\mathrm{HEMA}]_{i}=15 \%(\mathrm{~V} / \mathrm{V})$ by irradiation in absence of air, during 10 and $30 \mathrm{~h}$, respectively, at a dose-rate of $0.3 \mathrm{kGy} \mathrm{h}^{-1}$.

\subsection{Thermal analysis}

Figs. 3 and 4 show the weight loss curves of dry particle samples of LDPE, polyHEMA and PE-g-
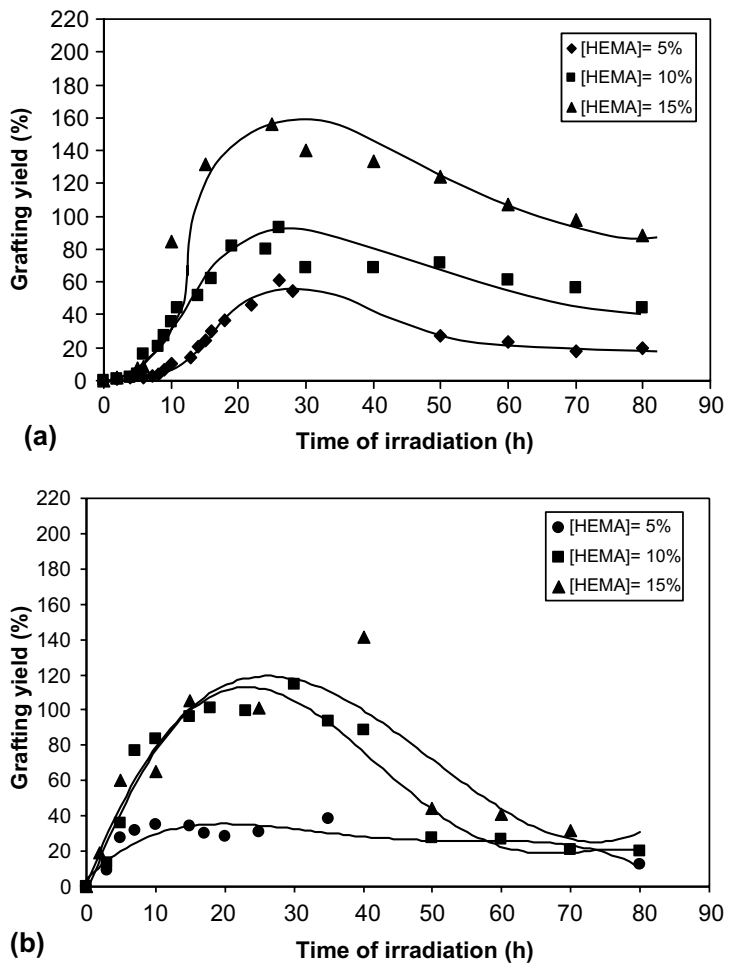

Fig. 2. Effect of [HEMA $]_{i}$ on the grafting yield of HEMA onto LDPE (particles) at an average dose-rate of $0.5 \mathrm{kGy} \mathrm{h}^{-1}$ : (a) gamma irradiation in presence of air; (b) gamma irradiation in absence of air.

HEMA samples with different grafting yields obtained by irradiation both in the presence and the absence of air at a dose-rate of $0.3 \mathrm{kGy} \mathrm{h}^{-1}$ with a $[\mathrm{HEMA}]_{i}=15 \%(\mathrm{~V} / \mathrm{V})$, which correspond to the experimental conditions that lead to high grafting yields.

TGA curves obtained from LDPE show that after $30 \mathrm{~h}$ of irradiation at $0.3 \mathrm{kGy} \mathrm{h}^{-1}$, the initial thermal decomposition temperature is slightly higher than that obtained from non-irradiated samples: increase of $\cong 2{ }^{\circ} \mathrm{C}$ when irradiated in the presence of air and of $\cong 5{ }^{\circ} \mathrm{C}$ when irradiated in the absence of air. This is in agreement with reported behaviour of gamma irradiated LDPE; the irradiation in the absence of air leads to a low or inexistent oxidation of the LDPE chains, which may lead to the larger temperature difference observed $[5,6]$. 


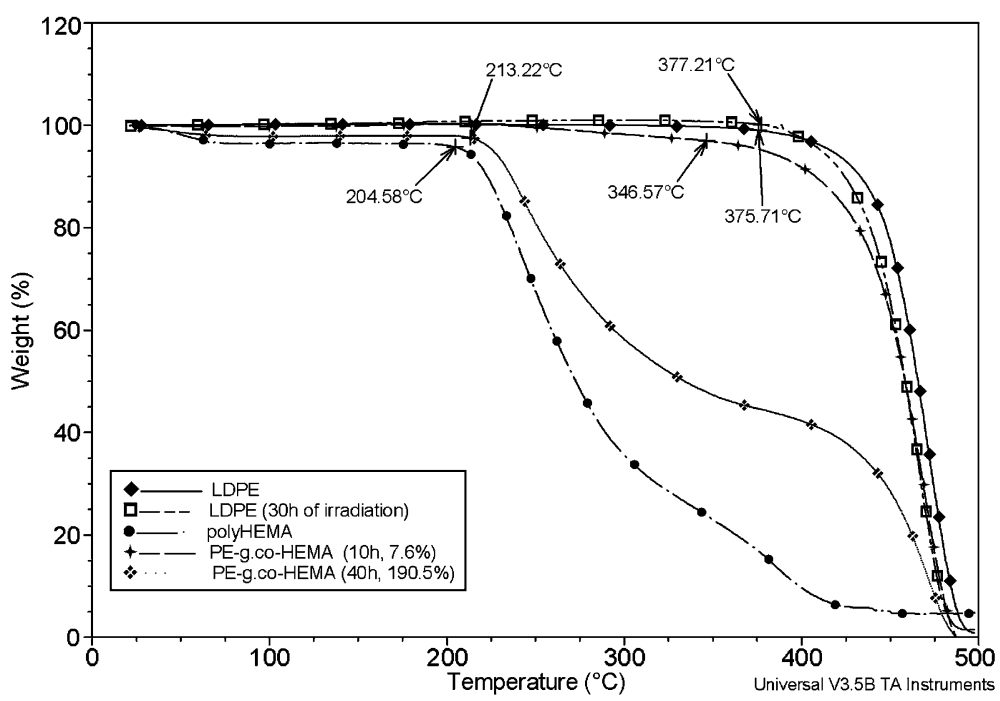

Fig. 3. TGA thermograms of particle samples of LDPE, LDPE irradiated in methanol, polyHEMA and PE-g-HEMA with different grafting yields, obtained by gamma irradiation in presence of air $\left(\mathrm{DR}=0.3 \mathrm{kGy} \mathrm{h}^{-1},[\mathrm{HEMA}]_{i}=15 \% \mathrm{~V} / \mathrm{V}\right)$.

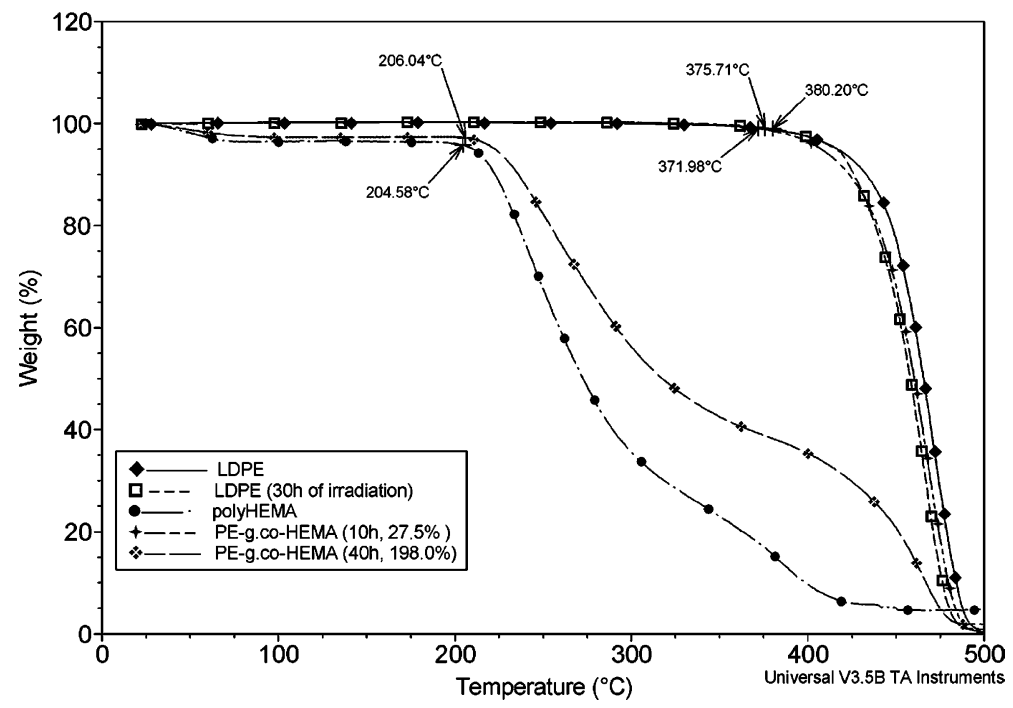

Fig. 4. TGA thermograms of particle samples of LDPE, LDPE irradiated in methanol, polyHEMA and PE-g-HEMA with different grafting yields, obtained by gamma irradiation in absence of air $\left(\mathrm{DR}=0.3 \mathrm{kGy} \mathrm{h}^{-1},[\mathrm{HEMA}]_{i}=15 \% \mathrm{~V} / \mathrm{V}\right)$.

It was also observed that, with increasing grafting yield, the thermal behaviour of the copolymers increasingly approaches that of polyHEMA, and departures from that of pure LDPE. This effect that has already been reported by other authors about the grafting of HEMA onto other polymers, suggests that the length of the grafted HEMA grows with the increase of grafting yield [4]. Nevertheless, above $300{ }^{\circ} \mathrm{C}$, the thermal behaviour of the copolymers approaches that of the LDPE backbone. That suggests that the copolymeric materials keep part of the LDPE structural identity.

This fact is in agreement with the thermal stability data obtained by DSC analysis (Table 1). 
Table 1

Endothermic transition temperature and melting enthalpy obtain by DSC analysis of particle samples of LDPE, and PE-g-HEMA with different grafting yields $\left(\mathrm{DR}=0.3 \mathrm{kGy} \mathrm{h}^{-1}\right.$, $\left[\mathrm{HEMA}_{i}=15 \%(\mathrm{~V} / \mathrm{V})\right)$

\begin{tabular}{lcll}
\hline Material & $\begin{array}{l}\text { Grafting } \\
\text { yield } \\
(\%)\end{array}$ & $\begin{array}{l}\text { Endothermic } \\
\text { transition } \\
\text { temperature }\left({ }^{\circ} \mathrm{C}\right)\end{array}$ & $\begin{array}{l}\text { Melting } \\
\text { enthalpy } \\
\left(\mathrm{J} \mathrm{g}^{-1}\right)\end{array}$ \\
\hline LDPE & 0 & 92.17 & 77.74 \\
PE-g-HEMA & $7.6^{\mathrm{a}}$ & 91.98 & 79.23 \\
& $190.5^{\mathrm{a}}$ & 93.17 & 38.87 \\
& $27.5^{\mathrm{b}}$ & 91.84 & 95.69 \\
& $198.0^{\mathrm{b}}$ & 93.54 & 41.45 \\
\hline
\end{tabular}

${ }^{a}$ Irradiated in presence of air.

b Irradiated in absence of air.

These data provides information about the changes in structural order of LDPE chains promoted by the graft copolymerisation reaction.

It can be observed that the endothermic transition temperature does not depart considerably from that of pure LDPE, irrespective of the grafting yield. It seems that the grafted polyHEMA act as a protective shield of the LDPE backbone, somehow preventing structural damage of the matrix. On the other hand the melting enthalpy of the grafted copolymers decreases with increasing yield of grafting, being the difference slightly higher when the irradiations are carried out in the presence of air. This behaviour suggests a disordering (loss of crystallinity) of the LDPE backbone promoted by the HEMA grafting [3]. The effect is, however, not dramatic.

Data from LDPE grafted films also show a clear departure from polyHEMA data. Fig. 5 shows results for films having grafting yields of $14.0 \%$ and $268.0 \%$. The curve for the sample grafted at $268.0 \%$ does not show the clear inflexion observed with particles. This is probably due to the difference in grafting yields.

\subsection{FTIR analysis}

The FTIR spectra of LDPE film, polyHEMA and prepared LDPE grafted films with 14.0\% and $268.0 \%$ of grafting, respectively are presented in Fig. 6. The infrared analysis was based on the identification of absorption bands related to vibrations of functional groups present in the molecules of polyHEMA and non-existent in LDPE molecules. These are the vibrations characteristics of the hydroxyl group $\left(v_{\mathrm{O}-\mathrm{H}}\right)$ from $3600-3200 \mathrm{~cm}^{-1}$,

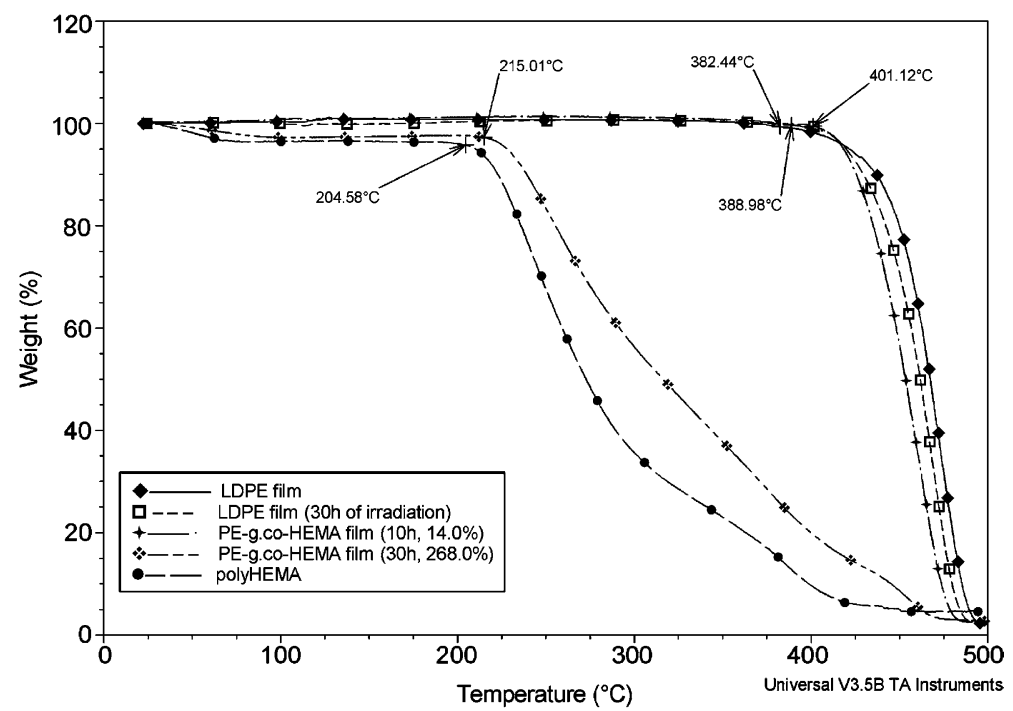

Fig. 5. TGA thermograms of LDPE film, LDPE film irradiated in methanol, polyHEMA and two PE-g-HEMA films with grafting yields of $14.0 \%$ and $268.0 \%$, obtained by gamma irradiation in absence of air $\left(\mathrm{DR}=0.3 \mathrm{kGy} \mathrm{h}^{-1},[\mathrm{HEMA}]_{i}=15 \% \mathrm{~V} / \mathrm{V}\right)$. 


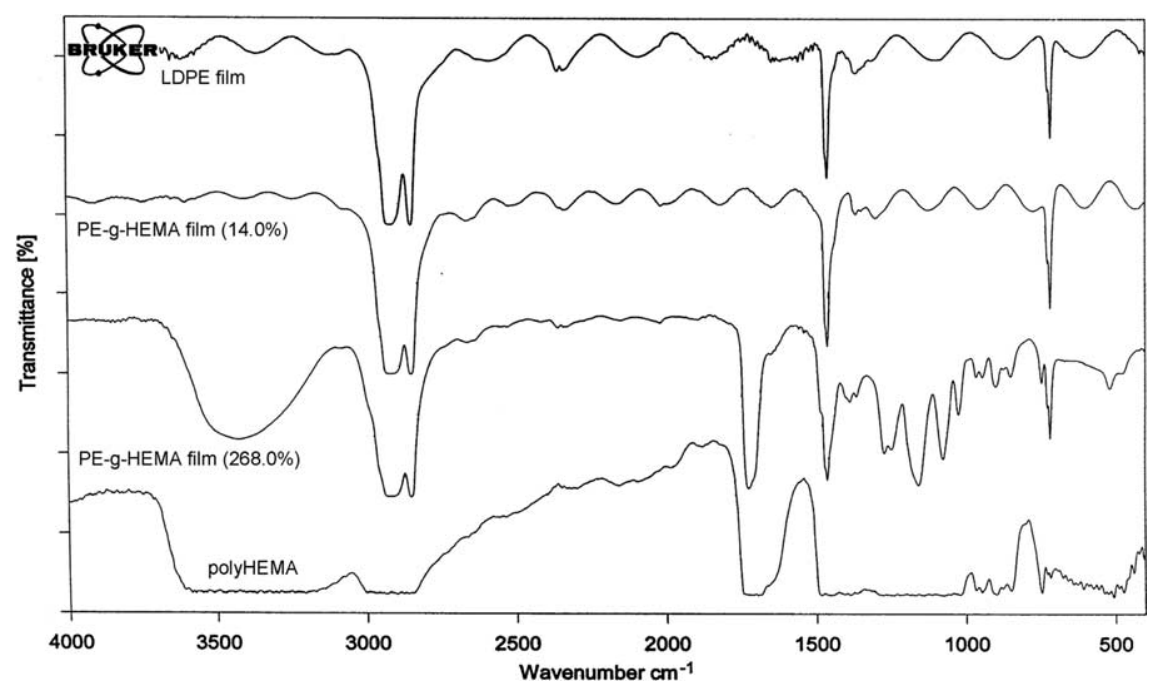

Fig. 6. IR spectra of LDPE film, polyHEMA and two PE-g-HEMA films with different grafting yields, obtained by gamma irradiation in absence of air $\left(\mathrm{DR}=0.3 \mathrm{kGy} \mathrm{h}^{-1},[\mathrm{HEMA}]_{i}=15 \% \mathrm{~V} / \mathrm{V}\right)$.

and those of the carbonyl group $\left(v_{\mathrm{C}}=\mathrm{O}\right)$ at $1735 \mathrm{~cm}^{-1}$ with a characteristic shoulder at $1650 \mathrm{~cm}^{-1}$.

The films studied belong to the region where grafting increases with dose. In this region, one observes a continuous increasing of the peaks characteristic of methacrylic polymers, with increasing grafting. These results again seem to confirm that, in this region of the yield versus dose plot, polyHEMA grafted branches grow with increasing degree of grafting.

\subsection{Hydration capacity of the grafted films}

The hydration capacity of a polymeric material to be used as a biomaterial is of major importance. The hydration degree influences the surface properties and the diffusion mechanisms through the polymeric support, as well as its mechanical properties [7].

Fig. 7 show the isothermal water absorption curves for LDPE grafted films as a function of time. Independently of the grafting degree both films studied attained their maximum hydration capacity ( $\cong 4 \%$ for PE-g-HEMA film with $14.0 \%$ of graft and $\cong 45.0 \%$ for PE-g-HEMA film with $268.0 \%$ of graft) in less than $2 \mathrm{~min}$.

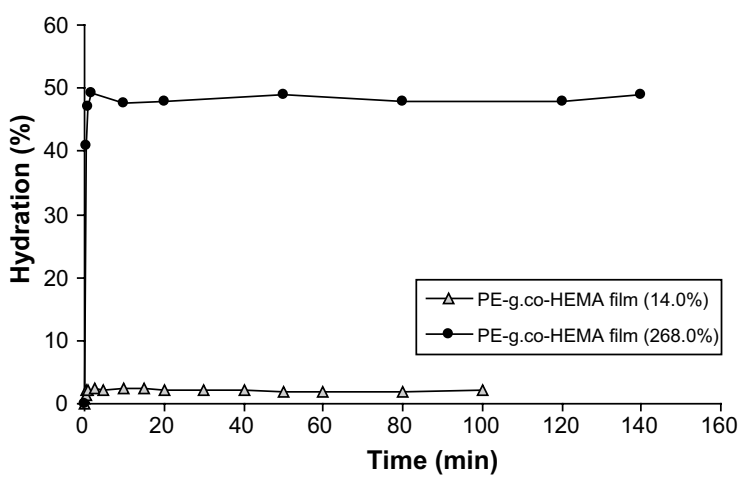

Fig. 7. Hydration capacity of LDPE grafted films at $37^{\circ} \mathrm{C}$ in physiological serum as function of time.

Isothermal dehydration data shows that, for the LDPE film grafted at $268.0 \%$, the dehydration kinetic is almost 7 times slower than that of the hydration kinetic. For the poor grafted film the kinetics ratio is almost 1:1 (Fig. 8).

The thickness of the films is clearly increased by the grafting of polyHEMA promoted by gamma radiation. Upon immersion in physiologic serum, the grafted films swell (Table 2).

These results show an improved hydrophilicity of the base material. 


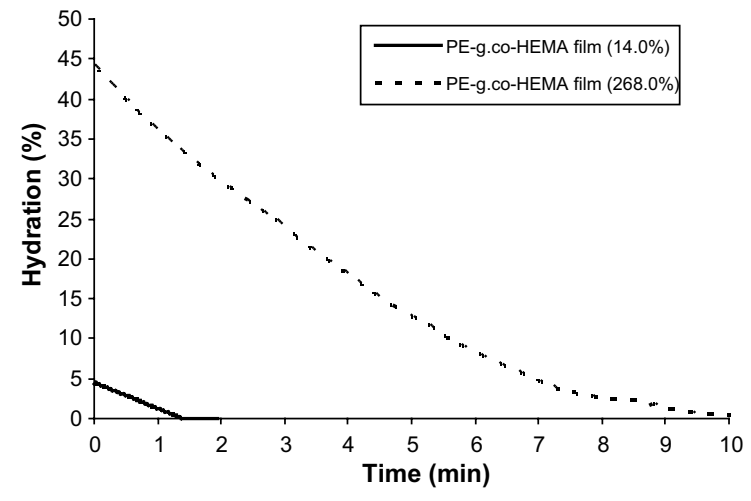

Fig. 8. Dehydration behaviour of LDPE grafted films at $37^{\circ} \mathrm{C}$ as function of time.

Table 2

Thickness of LDPE film before and after grafting process (dry and at hydration equilibrium)

\begin{tabular}{lcll}
\hline Material & Grafting yield $(\%)$ & \multicolumn{2}{l}{ Thickness $(\mu \mathrm{m})$} \\
\cline { 3 - 4 } & & Dry & Wet \\
\hline LDPE film & 0 & 15 & 15 \\
& 14.0 & 19 & 20 \\
& 268.0 & 42 & 94 \\
\hline
\end{tabular}

\section{Conclusions}

Graft copolymerisation induced by gamma radiation was shown to be a good technique for the preparation of new grafted copolymeric LDPE films. The results obtained show that the heterogeneous reactional system is sensitive to the irradiation environment, dose-rate and monomer concentration. The best results concerning grafting yield were obtained with low dose-rate, in the absence of air and with high monomer concentration.
The hydrophilic behaviour shown by the two copolymeric films prepared seems to be very promising. Taking as support LDPE, a hydrophobic polymer, it was obtained a final new material (PE-g-HEMA film) with improved hydrophilicity, which can reach a hydration level near $45.0 \%$, with a hydration/dehydration ratio of 1:7.

The modifications that occurred in the molecular structure of the copolymer backbone, namely some loss of crystallinity, do not seem to restrict its use, since the copolymer produced shows a good remaining thermal (and apparently mechanical) stability.

Future work on these systems will address the optimisation of graft films preparation, as well as the determination of other thermodynamic (e.g. glass transition temperature) and mechanical parameters. The shape of the grafting yield versus dose curve will be fully discussed in a forthcoming paper.

\section{References}

[1] F.W. Billmeyer, in: Textbook of Polymers Science, John Wiley \& Sons, Inc., New York, 1984.

[2] R.S. Benson, Nucl. Instr. and Meth. B 191 (2002) 752.

[3] L.M. Ferreira, J.M.S. Rocha, M.E. Andrade, M.H. Gil, Radiat. Phys. Chem. 52 (1998) 207.

[4] M.H. Casimiro, M.L. Botelho, J.P. Leal, M.H. Gil, Radiat. Phys. Chem. 72 (2005) 731.

[5] F.A. Bovey, in: The Effects of Ionizing Radiation on Natural and Synthetic High Polymers, Interscience Publishers, Inc., New York, 1958.

[6] F.A. Makhlis, in: Radiation Physics and Chemistry of Polymers, Halsted Press/John Wiley \& Sons, Inc., New York, 1975.

[7] C. Elvira, J.F. Mano, J.S. Román, R.L. Reis, Biomaterials 23 (2002) 1955. 